\title{
Prognosis of Pulmonary Embolism with Right Ventricular Dysfunction
} Alain Rougé ${ }^{1,2 *}$, Jérémie Lemarié ${ }^{1,2}$, Aurélie Cravoisy-Popovic ${ }^{1,2}$, Marie Conrad ${ }^{1,2}$, Lionel Nace ${ }^{1,2}$, Sébastien Gibot $^{1,2}$ and Pierre Edouard Bollaert ${ }^{1,2}$

${ }^{1}$ Medical Resuscitation Service, Central Hospital, Nancy University Hospital, Maréchal de Lattre Avenue Tassigny, Nancy, France ${ }^{2}$ University of Lorraine, Nancy, France

\begin{abstract}
Background: Mortality rate of pulmonary embolism (PE) at 3 months is over $15 \%$ for the high risk presentation and varies from $3 \%$ to $15 \%$ for the intermediate-risk presentation. Thrombolysis in intermediate-risk PE remains a matter of debate.
\end{abstract}

Methods: We undertook a retrospective study over a 11-year period including patients with high and intermediatehigh-risk PE, hospitalised in a medical ICU in a University hospital, to assess medium and long-term prognosis.

Results: Our series involved 145 patients, of whom 63 presented high risk PE and 82 had an intermediate-highrisk PE. Thirty-six patients (24.8\%) died in the intensive care unit (ICU) including high-risk PE: $34 / 63(53.9 \%)$, of whom 25 after inaugural cardiac arrest (CA); intermediate-high-risk PE: 2/82 (2.4\%). On multivariate analysis, high blood lactates (OR: 1.88; IC 95\% 1.18-3.02, p=0.0083), resuscitated CA (OR: 12.33; IC 95\% 1.1-137.4, p=0.041), low subaortic velocity time integral (VTI) (OR: 9.22, IC 0.38-0.81, $p=0.0024)$ were independent factors of in-ICU mortality. Twenty-seven patients died after ICU discharge. Echocardiographic checks were performed in 63 patients, in which 4 (6.3\%) presented a chronic thromboembolic pulmonary hypertension (CTPH).

Conclusion: High-risk PE still has a high mortality rate in the ICU especially when revealed by a cardiac arrest. High blood lactates, resuscitated CA, and low subaortic VTI were independent predictive factors of mortality. In our retrospective cohort, long-term prognosis was good with only 4 cases of CTPH. The low mortality observed with anticoagulant-only treatment do not encourage thrombolysis for management of intermediate-high-risk PE patients.

Keywords: Pulmonary embolism; Right ventricular dysfunction; Thrombolysis; Chronic thromboembolic pulmonary hypertension

\begin{abstract}
Abbreviations: CA: Cardiac Arrest; CTPH: Chronic Thromboembolic pulmonary hypertension; ICU: Intensive Care unit; PE: Pulmonary Embolism; rtPA: Recombinant Tissue-type Plasminogen Activator; RVD: Right Ventricular Dysfunction; TTE: Transthoracic Echocardiogram; VTI: Velocity Time Integral
\end{abstract}

\section{Introduction}

Pulmonary embolism (PE) is a common cardiovascular disease, affecting 100 to 200 cases per 100,000 people every year [1]. The recommendations issued from the European society of cardiology (ESC) [2] and the American heart association (AHA) [3] have defined high-risk PE as PE complicated by cardiac arrest, shock state or persistent arterial hypotension; and intermediate-risk PE as PE with right ventricular dysfunction (RVD) but without arterial hypotension. RVD is a determining factor of the severity of PE and may be recognized from the levels of troponins, BNP (B-type natriuretic peptide) or Nt pro BNP [4], but is most accurately evaluated by a transthoracic echocardiogram [5,6]. High-risk PE, still has a mortality rate of over $15 \%$ within 3 months [7-9], intermediate-risk PE has a 3\% to $15 \%$ mortality rate within 3 months $[10,11]$. The recent ESC recommendations have stratified the therapeutic treatment of high-risk and intermediaterisk PE. Thrombolysis is the recommended treatment of high-risk PE $[12,13]$. However, still open to debate is the indication for thrombolysis in patients with intermediate-risk pulmonary embolism. As compared with heparin, the superiority and rapidity of thrombolysis on reperfusion, reversal of pulmonary arterial hypertension and RVD is now established $[14,15]$. However, meta-analyses did not suggest benefit of thrombolytic therapy for intermediate-risk PE, [14] and the two randomized studies conducted in patients with intermediaterisk PE displayed no mortality reduction in patients who received thrombolytics as compared with no thrombolytics [16,17]. We performed a retrospective study over an 11-year period covering all cases of high-risk and intermediate-high-risk pulmonary embolism with acute echocardiographic RVD, hospitalized in a medical ICU in a tertiary care hospital, in order to assess the medium and long-term prognosis. Finally, medical and echocardiographic monitoring was collected in all the patients after hospital discharge, in order to assess the chronic thromboembolic pulmonary hypertension rate.

\section{Materials and Methods}

Between July 2003 and July 2014, we retrospectively reviewed all the patients hospitalised for high-risk PE and intermediate-high-risk $\mathrm{PE}$ in the medical ICU at the Central hospital of Nancy University Hospital. We excluded all patients admitted for intermediate-lowrisk PE when biomarkers were elevated without RVD ultrasound on TTE. The data were collected on a VIVID 3 (General Electric Medical System) and Philips HDI 1500 cardiovascular ultrasound system. We collected the demographic descriptions, the medical history (history of thrombophlebitis of the lower limbs or PE, family history of thromboembolism, history of neoplasia, obesity, arterial hypertension, smoking, type II diabetes, dyslipidemia, chronic obstructive pulmonary disease, known thrombophilia), the clinical presentations, long-

*Corresponding author: Alain Rougé, Medical Resuscitation Service, Central Hospital, Nancy University Hospital, Maréchal de Lattre Avenue Tassigny, University of Lorraine, Nancy, France, Tel: +33372740000; E-mail: alain.rouge@hotmail.com

Received January 23, 2017; Accepted February 15, 2017; Published February 17, 2017

Citation: Rougé A, Lemarié J, Cravoisy-Popovic A, Conrad M, Nace L, et al. (2017) Prognosis of Pulmonary Embolism with Right Ventricular Dysfunction. J Cardiovasc Dis Diagn 5: 260. doi: 10.4172/2329-9517.1000260

Copyright: @ 2017 Rougé A, et al. This is an open-access article distributed under the terms of the Creative Commons Attribution License, which permits unrestricted use, distribution, and reproduction in any medium, provided the original author and source are credited. 
term treatments, ECGs, blood gases, troponins, BNP, transthoracic echocardiograms (TTE), and thoracic angiograms.

\section{Definitions}

According to the recommendations of the ESC (European Society of Cardiology) [2] and the AHA (American Heart Association) [3], $\mathrm{PE}$ was defined as presenting high-risk or intermediate risk. Highrisk PE was defined as PE complicated by cardiac arrest, shock state or persistent arterial hypotension (defined as systolic blood pressure $<90$ $\mathrm{mm} \mathrm{Hg}$, or a systolic pressure drop by $\geq 40 \mathrm{~mm} \mathrm{Hg}$, for 15 minutes, if not caused by new-onset arrhythmia, hypovolemia, or sepsis).

Intermediate-risk PE was defined as $\mathrm{PE}$ with right ventricular dysfunction but without arterial hypotension. Patients who display evidence of both RVD (by TTE) and elevated cardiac biomarker levels in the circulation (particularly a positive cardiac troponin test) were classified into an intermediate-high-risk category. Patients with isolated elevated biomarker testing without RVD at TTE examination were excluded.

Right ventricular dysfunction in TTE was defined as RV enlargement with a quotient of end-diastolic lateral-septal RV diameter/end-diastolic left ventricular lateral-septal diameter $>0.9$. Additional parameters were a paradoxical septal motion (defined as a movement of the interventricular septum away from the left ventricular free wall during systole), and pressure overload with tricuspid valve insufficiency $[3,18]$. Troponin I values were elevated if they exceeded $0.1 \mathrm{ng} / \mathrm{ml}$

We also studied the subaortic velocity time integral (VTI) in order to evaluate cardiac output and the circulatory consequences of pulmonary embolism. The subaortic VTI was defined low when less than $13 \mathrm{~cm} / \mathrm{sec}$. Normal value of subaortic VTI is $16 \mathrm{~cm} / \mathrm{sec} \pm 3 \mathrm{~cm} /$ $\sec [18,19]$.

Chronic thromboembolic pulmonary hypertension $(\mathrm{CTPH})$ was defined as an increase in mean pulmonary arterial pressure (PAP) $\geq 25$ $\mathrm{mmHg}$ at rest that persists 6 months after PE diagnosis [20]. CTPH is now a distinct subgroup of pulmonary hypertension (group 4) without attempting to distinguish between proximal and distal forms of pulmonary hypertension [21]

\section{Treatment Strategy}

Effective anticoagulation was started as soon as possible after clinical suspicion or diagnosis of $\mathrm{PE}$ and was at the discretion of the physician in charge of the patient (unfractionated heparin (UFH), low molecular weight heparin (LMWH), fondaparinux (FDX)). When UFH was chosen, a bolus of $50 \mathrm{IU} / \mathrm{Kg}$ was given followed by continuous administration, titrated for activated cephalin time (ACT) between 60 and 90 seconds. The indication for thrombolysis was decided upon in the event of persistent arterial hypotension (as exposed in definition section) associated with RVD. Thrombolytic treatment with recombinant tissue-type plasminogen activator (rtPA) (alteplase) was administered at a dose of $0.6 \mathrm{mg} / \mathrm{kg}$ for 15 minutes [22]. Anticoagulation was stopped during thrombolysis, then restarted once the value of ACT was below twice the upper limit of normal. To minimize the risk of haemorrhage, a low invasive approach was used by all physicians: no routine arterial or central venous lines, no pulmonary artery catheter was inserted during the acute phase.

\section{Monitoring}

During ICU stay, various types of treatment and equipment used

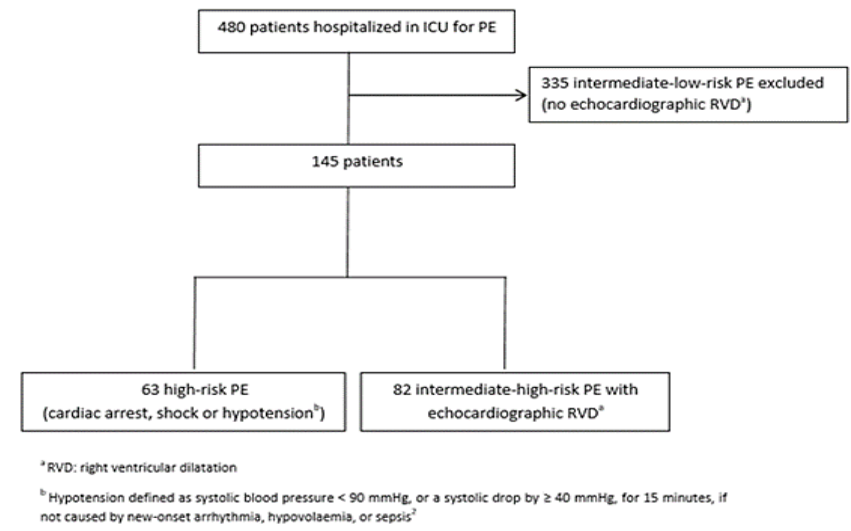

Figure 1: Flow chart.

were studied, as were organ failure replacement therapies. Every complication was recorded, especially haemorrhagic or neurological events and deaths; the length of hospital stay was also studied.

Medium and long-term monitoring was also performed retrospectively by studying each medical file, consultations and subsequent hospital admissions, and by calling upon GPs and cardiologists dealing with the patients. The subject of the requests was survival and thromboembolic relapses, the last control echocardiogram was systematically analysed, looking for signs of CTPH.

\section{Statistics}

Qualitative variables were analyzed using a $\chi^{2}$ test or a Fisher exact test and expressed as numbers and percentages (n, \%). Quantitative variables were analysed using non-parametric tests (Mann-Whitney or Kruskal-Wallis tests as appropriate), and expressed as medians (interquartile ranges). To determine prognostic factors of ICU mortality, a multiple stepwise logistic regression model was established with any covariate with univariate significance of $\mathrm{p}<0.10$ eligible for inclusion in the model which was further calibrated through HosmerLemeshow testing. To elucidate the effect of PE severity on mortality, Kaplan-Meier survival curves were constructed and analysed using a log-rank test. A value of $\mathrm{p}<0.05$ was deemed significant.

\section{Ethics}

The study was submitted to the "Comité de Protection des Personnes" which attested that it was a non-interventional retrospective study. According to the French law, patients received information about the study and a non-opposition to their participation in the study was sought.

\section{Results}

Between July 2003 and July 2014, we retrospectively reviewed 480 patients. As stated in the flow chart (Figure 1), the study included patients admitted for high-risk PE and intermediate-high-risk PE according to ESC guidelines [2]. Three hundred thirty-five patients who were admitted for intermediate-risk PE with isolated abnormal biomarker testing without RVD noted at TTE were excluded.

\section{Demographic Characteristics and Clinical Presentation}

Our series involved 145 patients, of whom 63 presented high risk PE and 82 had an intermediate-high-risk PE. The demographic characteristics are described in Table 1. Arterial hypertension was 
Citation: Rougé A, Lemarié J, Cravoisy-Popovic A, Conrad M, Nace L, et al. (2017) Prognosis of Pulmonary Embolism with Right Ventricular Dysfunction. J Cardiovasc Dis Diagn 5: 260. doi: 10.4172/2329-9517.1000260

Page 3 of 7

\begin{tabular}{|c|c|c|c|c|}
\hline Characteristics & Total $(n=145)$ & High-risk PE (n=63) & Intermediate-high-risk PE $(n=82)$ & $p$ \\
\hline Male sex & $46(31.7 \%)$ & $21(33 \%)$ & $25(30 \%)$ & 0.58 \\
\hline Median age (years) & $70(56-78)$ & $70(59-77)$ & $71(54-80)$ & 0.62 \\
\hline Previous deep vein thrombosis & $24(16.6 \%)$ & $9(14.3 \%)$ & $15(18.3 \%)$ & 0.52 \\
\hline Previous pulmonary embolism & $18(12.4 \%)$ & $8(12.7 \%)$ & $10(12.2 \%)$ & 0.93 \\
\hline Familial previous thromboembolism & $12(8.3 \%)$ & $3(4.8 \%)$ & $9(11.0 \%)$ & 0.18 \\
\hline Active cancer & $13(9.0 \%)$ & $10(15.9 \%)$ & $3(3.7 \%)$ & 0.01 \\
\hline Obesity & $14(9.7 \%)$ & $4(6.3 \%)$ & $10(12.2 \%)$ & 0.24 \\
\hline Hypertension & $76(52.4 \%)$ & $33(52.4 \%)$ & $43(52.4 \%)$ & 0.99 \\
\hline Tobacco use & $35(24.1 \%)$ & $15(23.8 \%)$ & $20(24.4 \%)$ & 0.93 \\
\hline Oral contraceptive pill & $7(4.8 \%)$ & $3(4.8 \%)$ & $4(4.9 \%)$ & 0.97 \\
\hline Diabetes & $16(11.0 \%)$ & $6(9.5 \%)$ & $10(12.2 \%)$ & 0.61 \\
\hline Dyslipidemia & $35(24.1 \%)$ & $13(20.6 \%)$ & $22(26.8 \%)$ & 0.38 \\
\hline Chronic obstructive pulmonary disease & $6(4.1 \%)$ & $4(6.3 \%)$ & $2(2.4 \%)$ & 0.24 \\
\hline
\end{tabular}

Table 1: Characteristics of the patients at baseline.

\begin{tabular}{|c|c|c|c|c|}
\hline Characteristics & Total $(n=145)$ & High-risk PE (n=63) & Intermediate-high-risk PE $(n=82)$ & p \\
\hline Resuscitated cardiac arrest & $32(22.1 \%)$ & $31(49.2 \%)$ & $1(1.2 \%)$ & l \\
\hline Resuscitated inaugural cardiac arrest & $28(19.3 \%)$ & $28(44.4 \%)$ & $0(0.0 \%)$ & I \\
\hline Cardiogenic shock & $62(42.8 \%)$ & $62(98.4 \%)$ & $0(0.0 \%)$ & 1 \\
\hline $\mathrm{pH}$ & $7.39(7.29-7.45)$ & $7.29(7.15-7.37)$ & $7.43(7.39-7.46)$ & $<0.0001$ \\
\hline Plasma bicarbonate (mmol/L) & $19(16-21)$ & $17(14-18.5)$ & $21(18-23)$ & $<0.0001$ \\
\hline Plasma lactate $(\mathrm{mmol} / \mathrm{L})$ & $2.6(1.4-5.8)$ & $5(2.4-9.5)$ & $1.8(1.1-2.6)$ & $<0.0001$ \\
\hline$D$-dimer $(\mu \mathrm{g} / \mathrm{L})$ & $5556(4000-9624)$ & $7730(4486-10000)$ & $5000(2680-6200)$ & 0.0064 \\
\hline Troponin I (ng/mL) & $0.19(0.07-0.71)$ & $0.24(0.09-1.04)$ & $0.19(0.06-0.61)$ & 0.45 \\
\hline $\mathrm{BNP}(\mathrm{pg} / \mathrm{ml})$ & $333(111-1124)$ & $852(75-1478)$ & $235(51-1058)$ & 0.81 \\
\hline Bilateral PE on CT scan & $88(60.7 \%)$ & $26(41.2 \%)$ & $62(75.6 \%)$ & $<0.0001$ \\
\hline Proximal PE on CT scan & $82(56.6 \%)$ & $24(38.1 \%)$ & $58(70.7 \%)$ & $<0.0001$ \\
\hline Paradoxical septal motion & $79(54.5 \%)$ & $47(74.6 \%)$ & $32(39.0 \%)$ & $<0.0001$ \\
\hline Systolic pulmonary artery pressures & $50(45-60)$ & $50(35-60)$ & $55(45-65)$ & 0.0057 \\
\hline Left ventricular ejection fraction (\%) & $50(50-60)$ & $50(40-50)$ & $60(50-60)$ & $<0.0001$ \\
\hline Sub aortic VTI (cm) & $14(10-18)$ & $10(8-13)$ & $17(14-19)$ & $<0.0001$ \\
\hline Right heart thrombus & $14(9.7 \%)$ & $7(11.1 \%)$ & $7(8.5 \%)$ & 0.6 \\
\hline Patent foramen ovale & $3(2.1 \%)$ & $1(1.6 \%)$ & $2(2.4 \%)$ & I \\
\hline Concomitant deep vein thrombosis & $69(47.6 \%)$ & $23(36.5 \%)$ & $46(56.1 \%)$ & 0.019 \\
\hline
\end{tabular}

Table 2: Clinical and paraclinical examinations at baseline.

\begin{tabular}{|c|c|c|c|c|}
\hline Characteristics & Total $(n=145)$ & High-risk PE (n=63) & Intermediate-high-risk $\mathrm{PE}(\mathrm{n}=\mathbf{8 2})$ & p \\
\hline Thrombolysis & $56(38.6 \%)$ & $56(88.9 \%)$ & $0(0.0 \%)$ & I \\
\hline Surgical embolectomy & $1(0.7 \%)$ & $0(0.0 \%)$ & $1(1.2 \%)$ & I \\
\hline Unfractionated heparin & $75(51.7 \%)$ & $50(79.4 \%)$ & $25(30.5 \%)$ & $<0.0001$ \\
\hline Low molecular weight heparin & $41(28.3 \%)$ & $3(4.8 \%)$ & $38(46.3 \%)$ & $<0.0001$ \\
\hline Fondaparinux & $19(13.1 \%)$ & $0(0.0 \%)$ & $19(23.2 \%)$ & I \\
\hline Endo-tracheal intubation & $35(24.1 \%)$ & $33(52.4 \%)$ & $2(2.4 \%)$ & I \\
\hline Norepinephrine & $18(12.4 \%)$ & $18(28.6 \%)$ & $0(0.0 \%)$ & 1 \\
\hline Epinephrine & $39(26.9 \%)$ & $39(61.9 \%)$ & $0(0.0 \%)$ & I \\
\hline Dobutamine & $6(4.1 \%)$ & $6(9.5 \%)$ & $0(0.0 \%)$ & I \\
\hline Dopamine & $3(2.1 \%)$ & $3(4.8 \%)$ & $0(0.0 \%)$ & 1 \\
\hline Inhaled nitric oxide & $29(20.0 \%)$ & $25(39.7 \%)$ & $4(4.9 \%)$ & $<0.0001$ \\
\hline Central venous catheter & $2(1.4 \%)$ & $2(3.2 \%)$ & $0(0.0 \%)$ & I \\
\hline Intra-arterial catheter & $2(1.4 \%)$ & $2(3.2 \%)$ & $0(0.0 \%)$ & I \\
\hline Dialysis catheter & $9(6.2 \%)$ & $9(14.3 \%)$ & $0(0.0 \%)$ & 1 \\
\hline Pulmonary artery catheter & $1(0.7 \%)$ & $1(1.6 \%)$ & $0(0.0 \%)$ & 1 \\
\hline Acute renal failure & $25(17.2 \%)$ & $19(30.2 \%)$ & $6(7.3 \%)$ & $<0.0001$ \\
\hline Renal replacement therapy & $9(6.2 \%)$ & $9(14.3 \%)$ & $0(0.0 \%)$ & 1 \\
\hline Bleeding complications & $5(3.4 \%)$ & $5(7.9 \%)$ & $0(0.0 \%)$ & 1 \\
\hline Death in the intensive care unit & $36(24.8 \%)$ & $34(53.9 \%)$ & $2(2.4 \%)$ & I \\
\hline
\end{tabular}

Table 3: Intensive care treatment. 
Citation: Rougé A, Lemarié J, Cravoisy-Popovic A, Conrad M, Nace L, et al. (2017) Prognosis of Pulmonary Embolism with Right Ventricular Dysfunction. J Cardiovasc Dis Diagn 5: 260. doi: 10.4172/2329-9517.1000260

Page 4 of 7

\begin{tabular}{|c|c|c|c|}
\hline Characteristics & Death $(n=36)$ & Survival $(n=109)$ & p \\
\hline $\mathrm{pH}$ & $7.18(7.11-7.36)$ & $7.41(7.37-7.47)$ & $<0.0001$ \\
\hline Systolic blood pressure $(\mathrm{mmHg})$ & $85(80-90)$ & $120(90-140)$ & $<0.0001$ \\
\hline S1Q3 ECG aspect & $4(11.1 \%)$ & $46(42.2 \%)$ & 0.004 \\
\hline Plasma lactate $(\mathrm{mmol} / \mathrm{L})$ & $8(4-17)$ & $2.3(1.2-3.1)$ & $<0.0001$ \\
\hline Troponin I (ng/mL) & $0.44(0.07-1.16)$ & $0.17(0.07-0.49)$ & 0.12 \\
\hline Sub-aortic VTI (cm) & $8(8-10)$ & $16(13-18)$ & $<0.0001$ \\
\hline Right heart thrombus & $3(8.3 \%)$ & $11(10.1 \%)$ & 0.76 \\
\hline Concomitant deep vein thrombosis & $12(33.3 \%)$ & $57(52.3 \%)$ & 0.048 \\
\hline Proximal pulmonary embolism & $10(27.8 \%)$ & $82(75.2 \%)$ & 0.0001 \\
\hline Thrombolysis & $28(77.8 \%)$ & $26(23.9 \%)$ & $<0.0001$ \\
\hline Resuscitated cardiac arrest & $27(75.0 \%)$ & $5(4.6 \%)$ & $<0.0001$ \\
\hline Resuscitated inaugural cardiac arrest & $14(38.9 \%)$ & $4(3.7 \%)$ & $<0.0001$ \\
\hline Cardiogenic shock & $35(97.2 \%)$ & $27(24.8 \%)$ & $<0.0001$ \\
\hline Endo-tracheal intubation & $27(75.0 \%)$ & $8(7.3 \%)$ & $<0.0001$ \\
\hline Norepinephrine & $6(16.7 \%)$ & $12(11.0 \%)$ & 0.37 \\
\hline Epinephrine & $28(78.8 \%)$ & $11(11.1 \%)$ & $<0.0001$ \\
\hline Inhaled nitric oxide & $13(36.1 \%)$ & $16(14.7 \%)$ & 0.005 \\
\hline Renal replacement therapy & $13(36.2 \%)$ & $12(11.0 \%)$ & 0.0005 \\
\hline
\end{tabular}

Table 4: Predictive factors of mortality at discharge from ICU (univariate analysis).

\begin{tabular}{|c|c|c|c|c|c|c|}
\hline Characteristics & Coef & Standard error & Chi 2 Test & $\mathbf{P}$ & $\operatorname{Exp}($ Coef) & $\mathbf{C l}$ \\
\hline Intercept & 4.95 & 3.93 & 1.59 & 0.20 & 142.51 & $0.06-316985.88$ \\
\hline Systolic blood pressure $(\mathrm{mmHg})$ & -0.01 & 0.03 & 0.30 & 0.57 & 0.98 & $0.91-1.04$ \\
\hline S1Q3 ECG aspect & -2.00 & 2.05 & 0.95 & 0.32 & 0.13 & $0.002-7.51$ \\
\hline Plasma lactate $(\mathrm{mmol} / \mathrm{L})$ & 0.63 & 0.24 & 6.97 & 0.008 & 1.88 & $1.17-3.02$ \\
\hline Sub aortic VTI low (cm) & -0.59 & 0.19 & 9.22 & 0.001 & 0.55 & $0.37-0.81$ \\
\hline Concomitant deep vein thrombosis & -2.35 & 1.48 & 2.25 & 0.11 & 0.09 & $0.005-1.73$ \\
\hline Resuscitated cardiac arrest & 251 & 1.23 & 4.16 & 0.041 & 12.32 & $1.10-137.41$ \\
\hline Proximal pulmonary embolism & 0.78 & 136 & 033 & 0.563 & 2.19 & $0.15-31.84$ \\
\hline
\end{tabular}

Table 5: Predictive factors of mortality at discharge from ICU (multivariate analysis).

the most frequently encountered comorbidity (52.4\%), 21 patients (14.5\%) were receiving long-term antiplatelet drugs, one patient had an history of thrombophilia. There was no significant difference in any demographic characteristic between high and intermediate-risk PE groups.

The different initial symptoms are detailed in Table 2. Thirtytwo patients $(20.7 \%)$ presented cardiac arrest (CA), of them 28 were inaugural and 4 happened while under anticoagulant therapy. Sixtytwo $(42.8 \%)$ patients developed cardiogenic shock. Troponin $(0.19$ $\mu \mathrm{g} / \mathrm{L}$, IQR: $0.07-0.71)$ and BNP (333 pg/ml, IQR: 111-1124) were measured in respectively 77 and 18 patients. The TTE displayed RVD in 144 patients (99.3\%). The only patient not presenting RVD on echocardiogram suffered a prehospital cardiac arrest, was then thrombolysed before hospital admission. Diagnosis was confirmed by CT scan. He was admitted in a post-anoxic coma followed by brain death. Ultrasonography criteria are detailed in Table 2, 47 patients (74.6\%) presented an initial paradoxical septum in the high-risk PE group against 32 (39\%) in the intermediate-high-risk PE group.

\section{Treatment Strategies and Intensive Care Outcome}

As displayed in Table 3, patients with intermediate-high-risk PE did not undergo organ replacement therapy or invasive procedures. Thirty-five patients underwent endotracheal intubation and mechanical ventilation, of whom 30 for a CA in the high-risk PE group. Only 2 patients received drugs via a central venous catheter. Nine patients (6.2\%) subsequently received renal replacement therapy. Thrombolysis was administered to 56 patients $(38.6 \%)$, seven patients $(4.8 \%)$ had a thrombolysis contraindication.
Thirty-six patients $(24.8 \%)$ died in the intensive care unit (ICU) (34/63 high-risk PE (53.9\%); 2/82 intermediate-high-risk PE (2.4\%)) (Figure 1). Among the 32 resuscitated CA, 25 CA were inaugural, and 27 patients $(84.3 \%)$ died in the ICU. When cardiac arrest patients were removed from the analysis of high-risk PE group, cardiogenic shock ICU mortality rate was $25 \%(8 / 32)$.

Otherwise, 5 patients (3.4\%) presented a haemorrhagic complication (4 patients with alteplase and one patient with UFH). Two patients had an ischemic stroke, one patient presented a brain death state, and 9 patients suffered a post-anoxic coma (6.2\%) in the high-risk PE group. A vena cava filter was inserted in 2 patients (1.4\%).

\section{Prognosis Factors in Univariate and Multivariate Analysis at Discharge from the Intensive Care Unit}

Univariate analysis is summarised on Table 4. On multivariate analysis (Table 5), high level of lactates (OR: 1.88; IC 95\% 1.18-3.02, $\mathrm{p}=0.0083$ ), the presence of recovered CA (OR: 12.33; IC 95\% 1.1-137.4, $\mathrm{p}=0.041$ ), and a low subaortic VTI (OR: 9.223, IC 0.38-0.81, $\mathrm{p}=0.0024$ ) were independent factors associated with an increased risk of mortality.

\section{Long-Term Follow-Up}

The median follow-up was 1907 days (1119-3163). Fourteen patients were lost to follow-up (9.7\%). Twenty-seven patients died during follow-up, 9 in high-risk PE group and 18 in intermediatehigh-risk PE group ( $\mathrm{p}=0.24)$, after a median time of 1118 days (5741935). One patient died at three months and another one at one year in intermediate-high-risk PE group. Mortality rates at three months and one year were respectively $3.6 \%$ and $4.9 \%$ in this group. In high- 


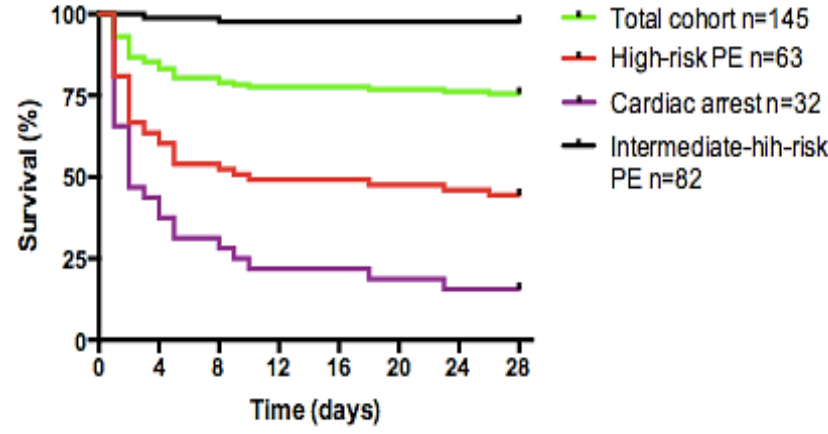

Figure 2: Kalpan-Meier 28 days survival curve.

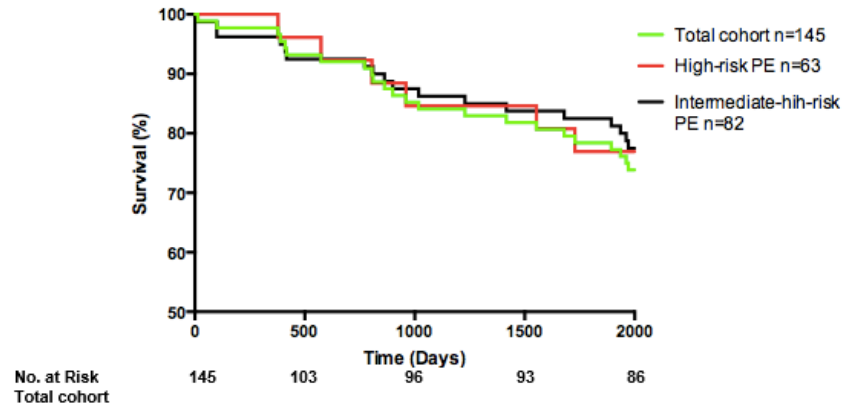

Figure 3: Kaplan-Meier Long term follow-up survival curve.

risk PE group, we did not find any new death in the first year followup period. The long-term follow-up survival curve (Figure 2) shows a superposition of mortality curves in each group.

Amongst the patients followed-up, we found only $4 \mathrm{PE}$ recurrences (2.8\%), all in the intermediate-high-risk group. Median of PE recurrence was 830 days (584-1125). Finally, echocardiogram controls using TTE were performed in 63 patients. Fifty-six TTE found normalization of cardiac function (88.9\%). Seven patients presented chronic pulmonary hypertension $(\mathrm{CPH})$, but it should be noted that three of them were suffering from chronic respiratory failure which could explain the $\mathrm{CPH}$. Four patients (6.3\%) including two patients in each group, had isolated chronic thromboembolic pulmonary hypertension (CTPH).

\section{Discussion}

The overall mortality rates of our series were $24.8 \%$ at 28 days and $25.5 \%$ at 3 months, both consistent with previous data [7-9,23]. We observed in this cohort that high-risk pulmonary embolism still has a high mortality rate especially when cardiac arrest occurs. Conversely, a very low mortality rate was observed in the intermediate-high-risk PE group managed with a conservative treatment excluding thrombolytic drugs. Finally, the long-term prognosis was good overall with only 4 recurrences, and 4 patients with CTPH.

Mortality in the high-risk PE group was $53.9 \%$ after 28 days, remaining the same after 3 months (Figure 3 ) in our series. This documented high-risk PE mortality rate at three months varies from $52.4 \%$ [8] to $58.3 \%$ [9]. The presence of inaugural cardiac arrest was the most important factor of mortality. Among the 32 patients who recovered from CA, 27 patients $(84.3 \%)$ died in the ICU. This mortality rate after CA varies according to studies, from $65 \%[7,24]$ to $90 \%$ [25]. When removing patients with CA from high-risk PE analysis, mortality rate in the ICU was $25 \%$. Our study showed the advantage of a conservative, standardised approach with the general principle of noninvasiveness as treatment of high-risk PE. Only 2 patients in our cohort received treatment through venous central line and underwent radial artery catheterization. Five patients required orotracheal intubation for another cause than cardiac arrest. Every patient who underwent thrombolysis received the same protocol and arterial blood samples were as far as possible avoided within the first 24 hours. This minimally invasive approach is consistent with the low rate of haemorrhagic complications observed (7.9\%), while varying in reports from $10.5 \%$ [9] to $17.6 \%$ [8]. Moreover, the rate of haemorrhagic accident in the high-risk PE group was $1.6 \%$ in our cohort, $2 \%$ in the PEITHO study (Pulmonary Embolism Thrombolysis) [17], and varies in the literature from $1.8 \%$ [26] to $2.2 \%$ [27].

Three-month mortality rates in intermediate-high-risk PE varies, depending on studies from $3 \%$ to $15 \%$ [10,11]. Our three months and one year mortality rates in the intermediate-high-risk PE group are low (respectively $3.6 \%$ and $4.9 \%$ ), thus do not encouraging thrombolysis for this subgroup of patients. The low mortality of our intermediatehigh-risk PE group is associated with no bleeding (Table 3). As a comparison, the rates of major and minor haemorrhagic complications were $11.5 \%$ and $32.6 \%$ respectively in the tenecteplase group in the PEITHO study [17] and the level of major hemorrhagic complications was $0.8 \%$ in the alteplase group from the study previously conducted by Konstantinides [16]. Our results reinforce this traditional attitude described as conservative at the expense of modern endovascular catheter-directed techniques aimed at reducing the systemic risk of haemorrhage [28]. Thrombolysis seems too dangerous in intermediatehigh-risk PE when compared with a strategy with anticoagulation and non-invasive monitoring in the ICU.

The subaortic VTI is a central parameter in echocardiographic haemodynamic evaluation in ICU. In our study, a low subaortic VTI was an independent prognostic factor of mortality in ICU $(p=0.001)$. The subaortic VTI is a major determinant of systolic ejection volume and is used to calculate cardiac output after measuring the left ventricle outflow tract diameter $\left[\mathrm{Q}=\mathrm{S}\left(\mathrm{cm}^{2}\right) \times\right.$ VTI $(\mathrm{cm}) \times$ FC $\left.\left(\mathrm{min}^{-1}\right)\right]$. In the most severe presentation of PE, RVD is accompanied by a reduced right ventricular systolic ejection volume; the secondary reduction in left ventricular preload may then lead to a reduction in cardiac output. Consequently, this parameter may be useful during TTE assessment of PE severity.

Finally, the long-term prognosis is good overall with only 4 recurrences, and 4 patients with chronic thromboembolic pulmonary hypertension (CTPH). Echocardiograms performed during follow-up showed a complete recovery in about $90 \%$ of patients. Although the incidence of CTPH is not definitely established, its prevalence varies according to studies from $0.8 \%$ [29] to $8.8 \%$ [30,31]. The risk factors for CTPH are the presence of repeated PE, the age and degree of pulmonary vascular obstruction [32]. Pulmonary hypertension $>60$ mmHg accompanied by a diagnosis of PE could be associated with an increased risk of persistent pulmonary hypertension after a year [31,33]. However, CTPH remains the only form of pulmonary hypertension which can be cured using pulmonary endarterectomy [34].

Our study shows the limitations of a single unit retrospective study. Validated biomarkers of RVD during PE (i.e. tropo and BNP) were not screened in every patients. Otherwise, owing to the relatively low number of events, the multivariate model may be overfitted. Furthermore, right heart thrombus $(9.7 \%)$ was not a prognostic factor in our study, probably fostered by statistical power bias. However, the single centre aspect of this extensive cohort reinforces the uniform 
procedures of both diagnosis and treatment, including indication for thrombolysis. Finally, our work focus a population treated before the publication of the recent ESC recommendations which clearly detail the treatment of high and intermediate risk $\mathrm{PE}$.

\section{Conclusion}

In spite of modern diagnostic and therapeutic strategies, highrisk pulmonary embolism [35-37] still has a high mortality rate in the intensive care unit, especially when cardiac arrest occurred. High blood lactates, resuscitated cardiac arrest, and low subaortic Velocity Time Integral on echocardiogram [38] were independent predictive factors of mortality. During follow-up we found only 4 patients with chronic thromboembolic pulmonary hypertension. Finally, the low mortality observed with anticoagulant-only treatment do not encourage thrombolysis for management of intermediate-high-risk PE patients.

\section{Acknowledgements}

We gratefully acknowledge the valuable contribution of the Department of Intensive care unit of the CHU of Nancy, and the Faculty of Medicine of Nancy.

\section{Conflicts of Interest}

All authors declare that they have no conflict of interests.

\section{References}

1. Cohen AT, Agnelli G, Anderson FA, Arcelus JI, Bergqvist D, et al. (2007) Venous thromboembolism (VTE) in Europe. The number of VTE events and associated morbidity and mortality. Thromb Haemost 98: 756-764.

2. Konstantinides SV, Torbicki A, Agnelli G, Danchin N, Fitzmaurice D, et al (2014) ESC guidelines on the diagnosis and management of acute pulmonary embolism. Eur Heart J 35: 3033-69, 3069a-k.

3. Jaff MR, McMurtry MS, Archer SL, Cushman M, Goldenberg N, et al. (2011) Management of massive and submassive pulmonary embolism, iliofemora deep vein thrombosis, and chronic thromboembolic pulmonary hypertension: A scientific statement from the American Heart Association. Circulation 123 1788-17830

4. Sanchez O, Trinquart L, Caille V, Couturaud F, Pacouret G, et al. (2010) Prognostic factors for pulmonary embolism: the prep study, a prospective multicenter cohort study. Am J Respir Crit Care Med 181(2): 168-173.

5. Sanchez O, Trinquart L, Planquette B, Couturaud F, Verschuren F, et al. (2013) Echocardiography and pulmonary embolism severity index have independent prognostic roles in pulmonary embolism. Eur Respir J 42: 681-688.

6. Vanni S, Nazerian P, Pepe G, Baioni M, Risso M, et al. (2011) Comparison of two prognostic models for acute pulmonary embolism: clinical vs. righ ventricular dysfunction-guided approach. J Thromb Haemost 9(10): 1916-1923.

7. Kasper W, Konstantinides S, Geibel A, Olschewski M, Heinrich F, et al. (1997) Management strategies and determinants of outcome in acute major pulmonary embolism: Results of a multicenter registry. J Am Coll Cardiol 30: 1165-1171.

8. Kucher N, Rossi E, De Rosa M, Goldhaber SZ (2006) Massive pulmonary embolism. Circulation 113(4): 577-582.

9. Goldhaber SZ, Visani L, De Rosa M (1999) Acute pulmonary embolism Clinical outcomes in the international cooperative pulmonary embolism registry (ICOPER). Lancet 353: 1386-1389.

10. Grifoni S, Olivotto I, Cecchini P, Pieralli F, Camaiti A, et al. (2000) Short-term clinical outcome of patients with acute pulmonary embolism, normal blood pressure, and echocardiographic right ventricular dysfunction. Circulation 101(24): 2817-2822.

11. Frémont B, Pacouret G, Jacobi D, Puglisi R, Charbonnier B (2008) Prognostic value of echocardiographic right/left ventricular end-diastolic diameter ratio in patients with acute pulmonary embolism: Results from a monocenter registry of 1,416 patients. Chest 133(2): 358-362

12. Konstantinides S, Tiede N, Geibel A, Olschewski M, Just H (1998) Comparison of alteplase versus heparin for resolution of major pulmonary embolism. Am J Cardiol 82: 966-970.
13. Marti C, John G, Konstantinides S, Combescure C, Sanchez O, et al. (2015) Systemic thrombolytic therapy for acute pulmonary embolism: A systematic review and meta-analysis. Eur Heart J 36(10): 605-614.

14. Wan S, Quinlan DJ, Agnelli G, Eikelboom JW (2004) Thrombolysis compared with heparin for the initial treatment of pulmonary embolism: A meta-analysis of the randomized controlled trials. Circulation 110(6):744-9.

15. Sharifi M, Bay C, Skrocki L, Rahimi F, Mehdipour M, "MOPETT" Investigators (2013) Moderate pulmonary embolism treated with thrombolysis (from the "MOPETT" Trial). Am J Cardiol 111: 273-277.

16. Konstantinides S, Geibel A, Heusel G, Heinrich F, Kasper W (2002) Managemen strategies and prognosis of pulmonary embolism-3 trial investigators. Heparin plus alteplase compared with heparin alone in patients with submassive pulmonary embolism. N Engl J Med 347: 1143-1150.

17. Meyer G, Vicaut E, Danays T, Agnelli G, Becattini C, et al. (2014) Fibrinolysis for patients with intermediate-risk pulmonary embolism. N Engl J Med 370: 1402-1411.

18. Lang RM, Bierig M, Devereux RB, Flachskampf FA, Foster E, et al. (2006) Recommendations for chamber quantification. Eur J Echocardiogr 7(2): 79-108.

19. Lancellotti P, Price S, Edvardsen T, Cosyns B, Neskovic AN, et al. (2015) The use of echocardiography in acute cardiovascular care: Recommendations of the European Association of Cardiovascular Imaging and the acute cardiovascular care association. Eur Heart J Cardiovasc Imaging 16(2): 119-1146.

20. Galiè N, Hoeper MM, Humbert M, Torbicki A, Vachiery J-L, et al. (2009) Guidelines for the diagnosis and treatment of pulmonary hypertension: The Task force for the diagnosis and treatment of pulmonary hypertension of the european society of cardiology (ESC) and the European Respiratory Society (ERS), endorsed by the International Society of Heart and Lung Transplantation (ISHLT). Eur Heart J 30(20): 2493-2537.

21. Simonneau G, Gatzoulis MA, Adatia I, Celermajer D, Denton C, et al. (2013) Updated clinical classification of pulmonary hypertension. J Am Coll Cardio 62: D34-D41.

22. Sors H, Pacouret G, Azarian R, Meyer G, Charbonnier B, et al. (1994) Hemodynamic effects of bolus vs 2-h infusion of alteplase in acute massive pulmonary embolism: A randomized controlled multicenter trial. Chest 106 712-717.

23. Carson JL, Kelley MA, Duff A, Weg JG, Fulkerson WJ, et al. (1992) The clinical course of pulmonary embolism. N Engl J Med 326: 1240-1245.

24. Wu MY, Liu YC, Tseng YH, Chang YS, Lin PJ, et al. (2013) Pulmonary embolectomy in high-risk acute pulmonary embolism: the effectiveness of a comprehensive therapeutic algorithm including extracorporeal life support. Resuscitation 84: 1365-13670.

25. Kürkciyan I, Meron G, Sterz F, Janata K, Domanovits H, et al. (2000) Pulmonary embolism as a cause of cardiac arrest: Presentation and outcome. Arch Intern Med 160: 1529-1535

26. Kanter DS, Mikkola KM, Patel SR, Parker JA, Goldhaber SZ (1997) Thrombolytic therapy for pulmonary embolism. Frequency of intracranial hemorrhage and associated risk factors. Chest 111: 1241-1245.

27. Levine MN (1995) Thrombolytic therapy for venous thromboembolism. Complications and contraindications. Clin Chest Med 16(2): 321-328.

28. Pelliccia F, Schiariti M, Terzano C, Keylani AM, D'Agostino DC, et al. (2014) Treatment of acute pulmonary embolism: update on newer pharmacologic and interventional strategies. BioMed Res Int 2014: 410341.

29. Becattini C, Agnelli G, Pesavento R, Silingardi M, Poggio R, et al. (2006) Incidence of chronic thromboembolic pulmonary hypertension after a first episode of pulmonary embolism. Chest 130: 172-175.

30. Dentali F, Donadini M, Gianni M, Bertolini A, Squizzato A, et al. (2009) Incidence of chronic pulmonary hypertension in patients with previous pulmonary embolism. Thromb Res 124: 256-258

31. Guérin L, Couturaud F, Parent F, Revel MP, Gillaizeau F, et al. (2014) Prevalence of chronic thromboembolic pulmonary hypertension after acute pulmonary embolism. Prevalence of CTEPH after pulmonary embolism. Thromb Haemost 112: 598-605.

32. Pengo V, Lensing AWA, Prins MH, Marchiori A, Davidson BL, et al. (2004) Incidence of chronic thromboembolic pulmonary hypertension after pulmonary embolism. N Engl J Med 350: 2257-2264. 
Citation: Rougé A, Lemarié J, Cravoisy-Popovic A, Conrad M, Nace L, et al. (2017) Prognosis of Pulmonary Embolism with Right Ventricular Dysfunction. J Cardiovasc Dis Diagn 5: 260. doi: 10.4172/2329-9517.1000260

33. Ribeiro A, Lindmarker $P$, Johnsson $H$, Juhlin-Dannfelt A, Jorfeldt $L$ (1999) Pulmonary embolism: one-year follow-up with echocardiography doppler and five-year survival analysis. Circulation 99: 1325-1330.

34. Lang IM, Simonneau G, Pepke-Zaba JW, Mayer E, Ambrož D, et al. (2013) Factors associated with diagnosis and operability of chronic thromboembolic pulmonary hypertension. A case-control study. Thromb Haemost 110: 83-91.

35. Pruszczyk P, Goliszek S, Lichodziejewska B, Kostrubiec M, Ciurzyński M, et al. (2014) Prognostic value of echocardiography in normotensive patients with acute pulmonary embolism. JACC Cardiovasc Imaging. 7(6): 553-560.

36. Platz E, Hassanein AH, Shah A, Goldhaber SZ, Solomon SD (2012) Regional right ventricular strain pattern in patients with acute pulmonary embolism.
Echocardiogr Mt Kisco N 29(4): 464-470.

37. Lang RM, Badano LP, Mor-Avi V, Afilalo J, Armstrong A, et al. (2015) Recommendations for cardiac chamber quantification by echocardiography in adults: An update from the American Society of Echocardiography and the European Association of Cardiovascular Imaging. Eur Heart J Cardiovasc Imaging 16(3): 233-271.

38. Rudski LG, Lai WW, Afilalo J, Hua L, Handschumacher MD, et al. (2010) Guidelines for the echocardiographic assessment of the right heart in adults: A report from the American Society of Echocardiography endorsed by the European Association of Echocardiography, a registered branch of the European Society of Cardiology, and the Canadian Society of Echocardiography. J Am Soc Echocardiogr 23(7): 685-713. 